\title{
Status report of the Twente University Raman free electron laser
}

\author{
P.J.M. van der Slot \\ Nederlands Centrum voor Laser Research BV, Postbus 2662, 7500 CR Enschede, Netherlands \\ C. Penman and W.J. Witteman \\ Unversitent Twente, Faculteit der Technische Natuurkunde, Postbus 217.7500 AE Enschede, Netherlands
}

\begin{abstract}
We report successful operation of a Raman-type free electron laser situated at the University of Twente. It is based on a Marx generator, and uses a transmission line to produce a flat $(\Delta V / V \leq 2 \%) 500 \mathrm{kV}$ voltage pulse of 100 ns duration The parameters of the bifilar helical undulator are $\lambda_{\mathrm{u}}=3 \mathrm{~cm}, 0 \leq B_{\mathrm{u}} \leq 025 \mathrm{~T}$ The field emission diode and the undulator are immersed in an axial guiding field The radiation is guided using a circular waveguide with $8 \mathrm{~mm}$ inner radius We estimate the spontaneous power level, and give the calculated growth factor for a $\mathrm{TE}_{11}$ mode.
\end{abstract}

\section{Description of the experiment}

The Twente Raman FEL, configured as an amplıfier (see fig. 1), is built around a Marx generator and uses a coaxial transmission line as a pulse forming network to produce a flat voltage pulse of approxımately $500 \mathrm{kV}$ $\left(\gamma \cong 2\right.$ ). A low inductance gas switch filled with $\mathrm{SF}_{6}$ is used to connect the transmission line to the field emission diode (FED). A second coaxial transmission line with the same impedance $Z_{0}$ as the first is used to suppress the prepulse on the FED which appears during charging of the first transmission line. The matching resistor is in principle optional, but it has several advantages. It limits the duration of the voltage pulse to twice the transit tume of the first transmission line, i.e. $100 \mathrm{~ns}$, since the impedance of the FED, $Z_{\text {FtD }}$ is higher than $Z_{0}$. Also the voltage pulse is less sensitive to the change of the diode impedance caused by the expanding plasma in front of the cathode. A disadvantage of the matching resistor is that the voltage over the FED is only half the loading voltage of the transmission line.

The electron beam produced by the FED is apertured by a hole in the anode and injected into the cylindrical waveguide. The apertured current $I_{\mathrm{d}}$ is transported through the waveguide with the and of an axial guiding field which confines the beam. A pulsed, bifilar helical undulator with $N_{u}=40$ periods (the first seven periods form the tapered entrance) converts the kinetic energy of the electrons into radiation energy. This energy is coupled out of the system using a low-reflectance horn. An overview of relevant parameters of this system is shown in table 1.

\section{Spontaneous emission}

A free electron laser with axial guiding field produces two kinds of radiation which can both take part in stimulated processes: the transverse electron motion due to the undulator and the electron cyclotron motion about the axial magnetic field lines both produce oscillatory transverse currents, of different frequencies, which are capable of driving different modes of the laser field. The incoherent radiant intensity due to the undulator field $B_{\mathrm{u}}$ is

$\frac{\mathrm{d} P_{\mathrm{u}}}{\mathrm{d} \Theta}=\frac{e^{4}}{4 \pi \epsilon_{0} m^{2} c} \frac{\beta_{z}^{2} B_{\mathrm{u}}^{2}}{\left(1-\beta_{z}\right)^{3}} N_{\mathrm{e}}$.

where $N_{\mathrm{e}}$ is the number of electrons in the undulator. With our parameters, this takes a value of the order of 1 $W$ per steradian. For radiation due to cyclotron motion,

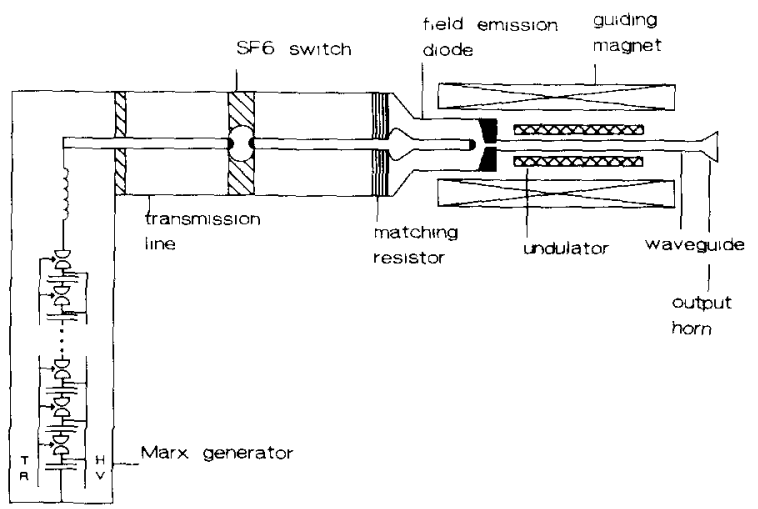

Fig. 1. Schematic layout of the Twente Raman free electron laser 
Table 1

An overview of the relevant parameters of the Twente Raman free electron laser

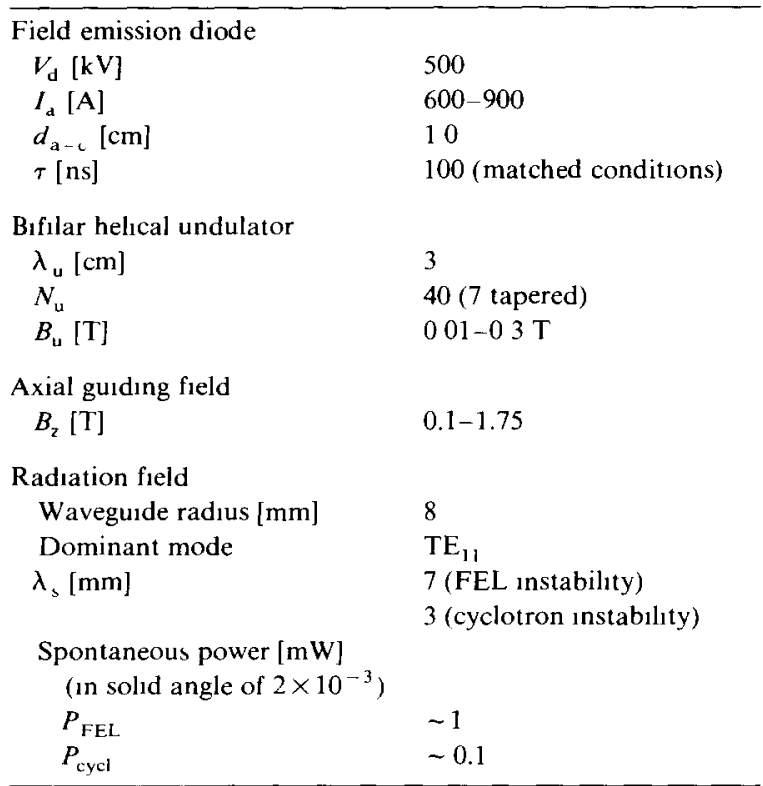

$\beta_{z} B_{\mathrm{u}}$ in the numerator of the above equation is replaced by $\beta_{\mathrm{T}} B_{z}$, where $c \beta_{\mathrm{T}}$ is the transverse velocity with which each electron enters the guiding field (considered here to be the same for all electrons). This takes a value $-0.1 \mathrm{~W}$ per steradian.

Feedback from the ECM field bunches the electrons azimuthally about the axial magnetic field lines, in a process similar to the normal FEL bunching mechanism. If the direction of the initial transverse motion is

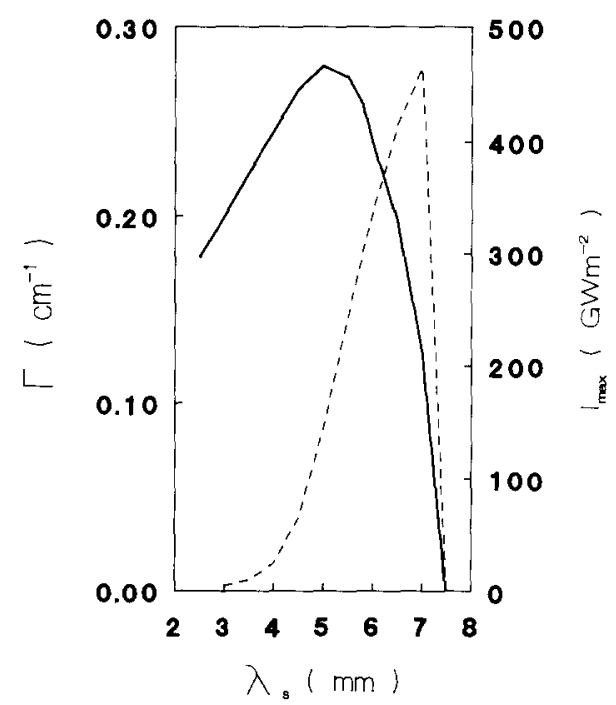

Fig. 2. The growth factor $\Gamma$ (solid line) and maximum on-axis intensity $I_{\max }$ (dashed curve) as a function of free-space wavelength $\lambda_{5}$ for a single $T E_{11}$ mode. Model parameters are $N_{\mathrm{u}}=40, \lambda_{\mathrm{u}}=3.0 \mathrm{~cm}, \gamma=2, I=750 \mathrm{~A}, a_{\mathrm{u}}=0.2$, and 100 electrons were used ( $a_{u}$ is the dimensionless undulator vector potentual).

correlated with transverse position, the stimulated ECM process can also be enhanced by longitudinal varıations in density [1]. The growth rate for the latter process can be comparable with that for FEL radiation [1].

\section{Stimulated emission}

Some preliminary calculations have been done to investigate stımulated emission. The model used [2] is
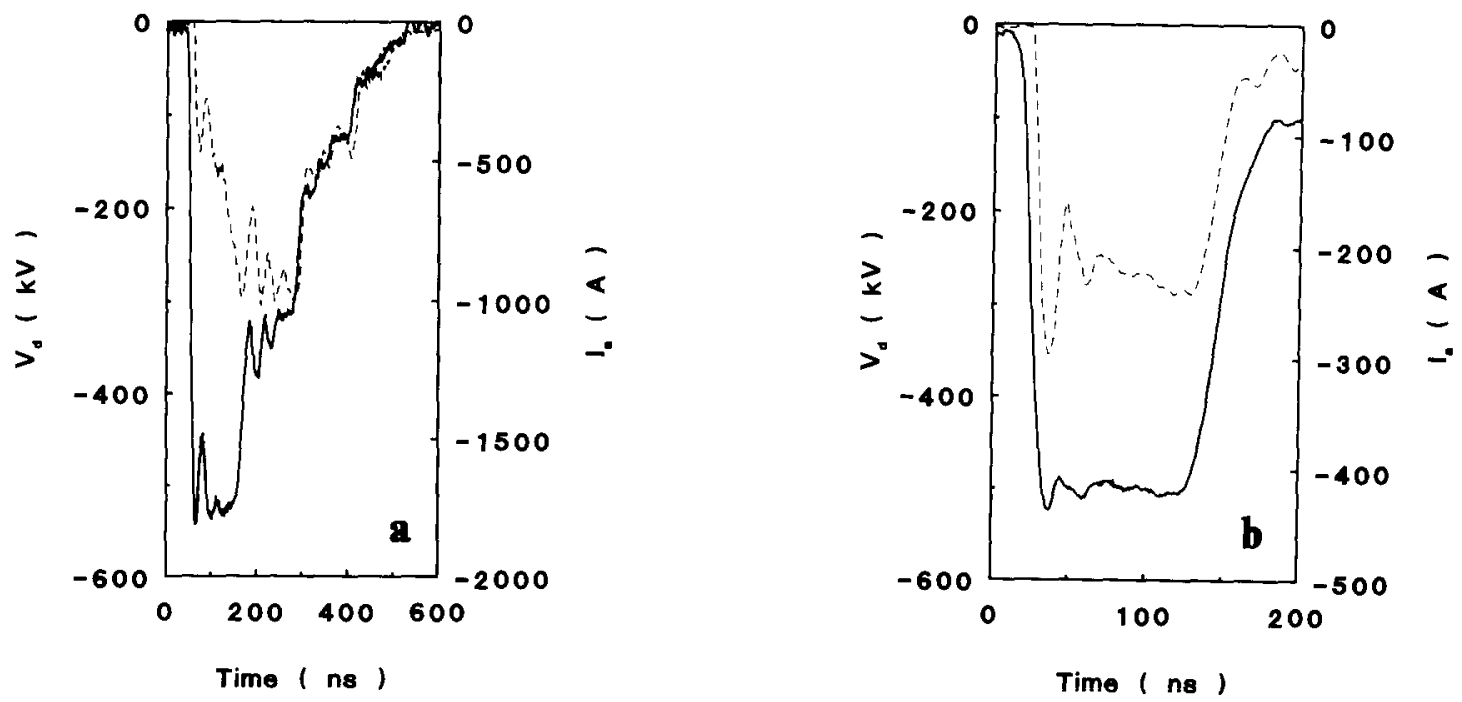

Fig. 3. Typical voltage $V_{\mathrm{d}}$ (solid line) over the field emission dıode and the apertured current $I_{\mathrm{a}}$ (dashed line) for (a) anode-cathode distance $d_{\mathrm{a}-\mathrm{c}}=1.0 \mathrm{~cm}$, no matching resistor, and (b) $d_{\mathrm{a}-\mathrm{c}}=1.5 \mathrm{~cm}$, matching resistor installed. 
one-dimensional in the electron equations and two-dimensional in the radiation freld. Thus it assumes that the scale of transverse electron motion is small compared with transverse variation of the field. In general we can treat the radiation field as a superposition of vacuum waveguide modes and investigate, e.g., optical guiding. For the calculations of the growth factor, however, we used only one mode, the dominant $\mathrm{TE}_{11}$ mode. In fig. 2 we plot the intensity growth rate, $\Gamma \equiv$ $\mathrm{d} / \mathrm{d} z\{\ln (I(z) / I(0))\}$, against the free-space wavelength $\lambda_{\mathrm{s}}$. Also the maximum on-axis intensity obtained in the undulator is plotted. It can be seen that although a large interval of wavelength shows exponential growth, only a relatively small interval contributes to the final energy in the pulse.

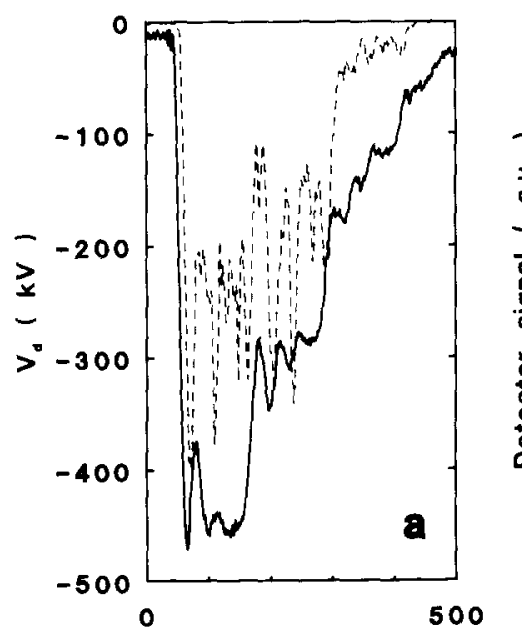

Time ( ns )

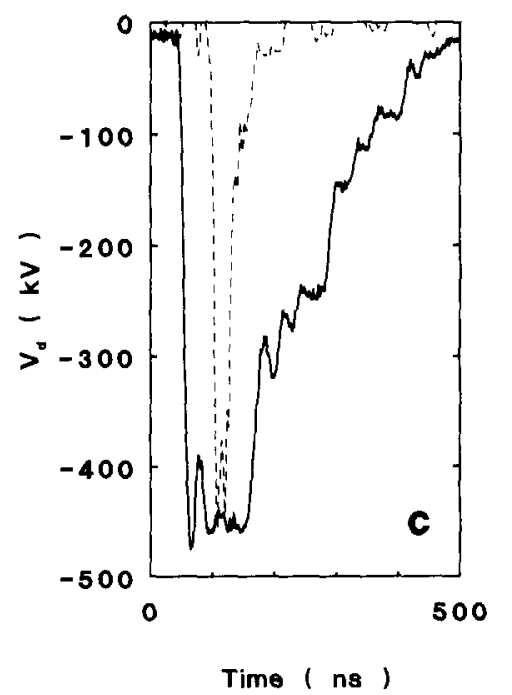

\section{Experimental results}

We are able to control the apertured current $I_{\mathrm{a}}$ by changing the guiding field strength or the anodecathode distance $d_{\mathrm{a}-\mathrm{c}}$. Both change $Z_{\text {FED }}$, thus changing the total current. It has to be noted that changing the guiding field influences the quality of the e-beam. In fig. 3 two typical shots are shown. The matching resistor was not present in fig. $3 \mathrm{a}$, where $d_{\mathrm{a}-\mathrm{c}}=1.0 \mathrm{~cm}$. Because of the high FED impedance, the voltage diminishes stepwise to zero. The oscillation at the beginnıng of each step is produced by an impedance mismatch at the connection of the first transmission line to the $\mathrm{SF}_{6}$ switch. The time-dependent behaviour of the FED is shown by the increase in $I_{\text {a }}$ due to the decrease of
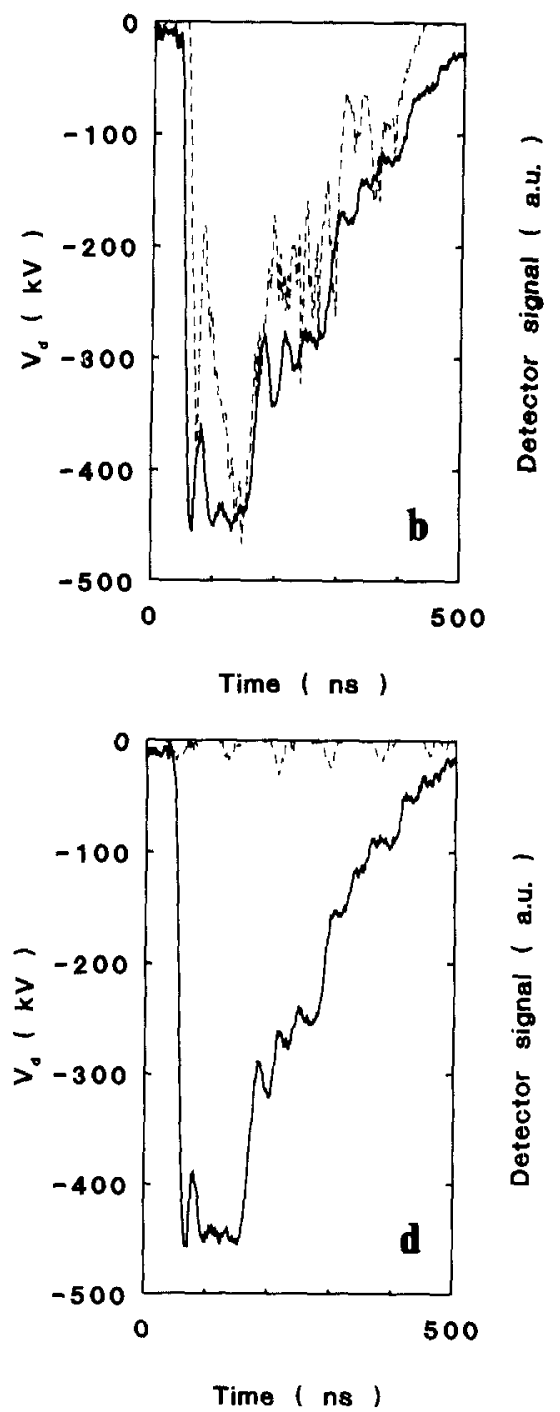

Fig. 4. Typical shots above magnetoresonance. Shown are the voltage $V_{\mathrm{d}}$ (solid line) over the field emission diode and the milımeter wave crystal detector signal (dashed curve) for the following combinations of guiding and undulator fields: (a) $B_{u}=0.18 \mathrm{~T}, B_{z}=1 \mathrm{~T}$, (b) $B_{\mathrm{u}}=0.28 \mathrm{~T}, B_{z}=1 \mathrm{~T}$, (c) $B_{\mathrm{u}}=0.28 \mathrm{~T}, B_{z}=03 \mathrm{~T}$, and (d) $B_{\mathrm{u}}=0 \mathrm{~T}, B_{z}=0.3 \mathrm{~T}$. 
$Z_{\text {FED }}$. At the end of the pulse a current of approximately $1 \mathrm{kA}$ is obtained. In fig. $3 \mathrm{~b}$ a matching resistor was installed and the oscillations at the beginning of the pulse have almost disappeared (note that the matching is not perfect). The change in $I_{d}$ is less than in fig. $3 \mathrm{a}$ because the fractional change in $Z_{\mathrm{FED}}$ is reduced by the larger value of $d_{\mathrm{a}-\mathrm{c}}=1.5 \mathrm{~cm}\left(Z_{\mathrm{FED}} \propto d_{\mathrm{a}-\mathrm{c}}^{2}\right)$. The current $I_{\mathrm{a}}$ dropped down to approximately $200 \mathrm{~A}$. From the time dependence of $Z_{\text {FED }}=V_{\mathrm{d}} / I_{\mathrm{t}}$, where $V_{\mathrm{d}}$ is the FED voltage and $I_{\mathrm{t}}$ the total current in the FED, we estimated the velocity of the expanding plasma in front of the cathode to be approxımately $1.1 \mathrm{~cm} / \mu \mathrm{s}$ at $V_{\mathrm{d}} \cong$ $500 \mathrm{kV}$. Fig. 3b shows that it is possible to produce a voltage pulse with $\Delta V_{\mathrm{d}} / V_{\mathrm{d}} \leq 1.5 \%$ for $80 \mathrm{~ns}$ if the FEL is operated under matched conditions, 1.e., the matching resistor is installed.

Very recently we have obtained successful operation of our FEL. Fig. 4 shows four typical shots done under unmatched conditions. In a FEL with an axial guiding field as well as an undulator one can distinguish two operatıng regimes, one below and the other above magnetoresonance [3]. For our experimental parameters magnetoresonance occurs near $B_{z}=0.62 \mathrm{~T}\left(B_{\mathrm{u}}=0\right)$, $0.26 \mathrm{~T}\left(B_{\mathrm{u}}=0.18 \mathrm{~T}\right)$ and $0.16 \mathrm{~T}\left(B_{\mathrm{u}}=0.28 \mathrm{~T}\right)$. Thus figs. $4 \mathrm{a}-4 \mathrm{c}$ apply to the regime above magnetoresonance. Calibration of the detector used (HP R442A crystal detector) has not yet been possible, but the measured signal levels are at least four orders of magnitude larger than the spontaneous power levels, which are $-1 \mathrm{~mW}$ at the position of the detector. In figs. $4 \mathrm{c}$ and $4 \mathrm{~d}$ all experimental parameters are kept constant except the strength of the undulator field which was turned off in the case of fig. $4 \mathrm{~d}$. The power levels and the difference between figs. $4 c$ and $4 d$ indicate that we have obtained stimulated FEL radiation. It is not possible to determine from figs. $4 \mathrm{a}$ and $4 \mathrm{~b}$ which part of the radiation is due to the FEL and which part to the cyclotron instability. Planned measurements of wavelength may give the answer.

The simulations mentioned above also show that saturation occurs well before the end of the undulator. This makes the interpretation of the measured signals as presented in fig. 4 difficult, so spatial growth and saturation will also be investigated in the near future.

\section{References}

[1] A. Fruchtman and L. Friedland, IEEE J. Quantum Electron. QE-19 (1983) 327.

[2] A. Bhattacharjee, S.Y. Ca1, S.P. Chang, J.W. Dodd, A. Fruchtman and T C. Marshall, Phys. Rev. A40 (1989) 5081.

[3] L. Friedland, Phys. Fluids 23 (1980) 2376 